\title{
Torcicolo congênito: avaliação de dois tratamentos fisioterapêuticos
}

\author{
Congenital torticollis: evaluation of two physiotherapeutic approaches
}

\section{Luciane Zanusso Pagnossim ${ }^{1}$, Augusto Frederico S. Schmidt ${ }^{2}$, Joaquim Murray Bustorff-Silva ${ }^{3}$, Sérgio Tadeu M. Marba ${ }^{4}$, Lourenço Sbragia ${ }^{5}$}

\section{RESUMO}

Objetivo: Avaliar a evolução clínica e comparar a eficácia do tratamento fisioterapêutico intensivo ou mínimo, em neonatos e lactentes com torcicolo congênito.

Métodos: Foram atendidas no Ambulatório de Fisioterapia e Cirurgia Pediátrica do Hospital de Clínicas da Universidade Estadual de Campinas 47 crianças com torcicolo congênito. Analisaram-se, retrospectivamente, antecedentes maternos, neonatais, apresentação clínica e tratamento fisioterapêutico, classificado de acordo com a frequiência das sessões. O tratamento intensivo foi definido como sessões de fisioterapia realizadas pelo fisioterapeuta no ambulatório, acrescidas de exercícios diários realizados pelos pais em domicílio. O tratamento mínimo foi considerado quando apenas o fisioterapeuta realizava as sessões semanalmente no ambulatório, sem a participação dos pais.

Resultados: Quanto às características demográficas da população, observou-se: idade média materna $=26$ anos; parto normal $=40(85 \%)$; mães primigestas $=35(75 \%)$; idade média ao diagnóstico $=50$ dias. A presença de nódulo intramuscular foi notada em $46(98 \%)$ pacientes. As seguintes doenças estiveram associadas: luxação congênita de quadril igual $=4(8 \%)$; fratura de clavícula $=2(4 \%)$; pé torto congênito $=1(2 \%)$. O tratamento intensivo foi realizado em $34(72 \%)$ pacientes e a cura observada em $100 \%$, após 74 dias, em média. O tratamento mínimo foi aplicado em 13 (28\%) pacientes e a cura observada em dez (77\%), tratados em média por 130 dias.

${ }^{1}$ Fisioterapeuta, mestre em Cirurgia pela Faculdade de Ciências Médicas da Universidade Estadual de Campinas (FCM-Unicamp). Campinas, SP, Brasil

${ }^{2}$ Mestrando em Cirurgia da FCM-Unicamp. Campinas, SP, Brasil

${ }^{3}$ Professor titular de Cirurgia Pediátrica do Departamento de Cirurgia da FCM-Unicamp. Campinas, SP, Brasil

${ }^{4}$ Professor adjunto da disciplina de Neonatologia do Departamento de Pediatria da FCM-Unicamp. Campinas, SP, Brasil

${ }^{5}$ Professor adjunto de Cirurgia Pediátrica do Departamento de Cirurgia da FCM-Unicamp. Campinas, SP, Brasil
Conclusões: $O$ torcicolo congênito apresentou evolução clínica favorável em ambos os grupos. O tratamento intensivo propiciou maior índice de cura em menor tempo de tratamento.

Palavras-chave: torcicolo congênito; fisioterapia; recémnascido; prognóstico.

\section{ABSTRACT}

Objective: To evaluate the clinical course and to compare the effectiveness of physiotherapeutic treatment performed daily or once a week in newborns and infants with congenital torticollis.

Methods: 47 patients with congenital torticollis were assisted at the Physiotherapy and Pediatric Surgery Services of the Clinical Hospital of Campinas University. The following data were retrospectively collected: maternal and neonatal history, clinical presentation and physiotherapeutic treatment classified according to the frequency of the exercises. Intensive treatment was defined as sessions performed at the daycare clinic by the physiotherapist plus daily sessions performed by parents at home. Minimal treatment was defined as weekly sessions performed only by the physiotherapist at the daycare clinic, without participation of the parents.

Results: The demographic characteristics of the studied patients were: mean maternal age $=26$ years; first gestation $=35(75 \%)$; vaginal delivery $=40(85 \%)$; mean age at diagnosis $=50$ days. A sternomastoid nodule was felt seen

Endereço para correspondência:

Lourenço Sbragia

Rua Alexander Flemming 181 - Cidade Universitária - Barão Geraldo

CEP 13083-970 - Campinas/SP

E-mail: sbragia@fcm.unicamp.br

Recebido em: 3/3/2008

Aprovado em: 12/5/2008

Fonte financiadora: Programa de Apoio à Pós-Graduação pela Coordenação de Aperfeiçoamento de Pessoal de Nível Superior (Capes) 
in $46(98 \%)$ patients. Diseases associated with congenital torticollis were: congenital hip dislocation $=4(8 \%)$; fracture of clavicles $=2(4 \%)$; and club feet $=1(2 \%)$. Intensive treatment was performed in $34(72 \%)$ patients for an average of 74 days and the cure was achieved in $100 \%$. Minimal treatment was performed in $13(28 \%)$ for an average of 130 days and ten $(77 \%)$ patients achieved cure.

Conclusions: Congenital torticollis had favorable outcome in both groups. The intensive treatment was followed by higher rates of cure in smaller period of time.

Key-words: congenital torticollis; physical therapy; infant newborn; prognosis.

\section{Introdução}

O torcicolo congênito (TC) é definido como uma contratura unilateral do músculo esternocleidomastoideo que, em geral, se manifesta no período neonatal ou em lactentes ${ }^{(1)}$. Em crianças com TC, a cabeça fica inclinada para o lado do músculo afetado e rodada para o lado oposto ${ }^{(2)}$. A sua incidência é de 1:250 recémnascidos $^{(3)}$ e a etiologia ainda não está esclarecida $a^{(2-4)}$.

As hipóteses relativas à etiologia da condição se relacionam ao tocotraumatismo cervical ${ }^{(5)}$, à isquemia arterial com hipofluxo sanguíneo para o esternocleidomastoideo ${ }^{(6,7)}$, à obstrução venosa do esternocleidomastoideo ${ }^{(8)}$, ao mal posicionamento intrauterino $^{(9)} \mathrm{e}$ à hereditariedade $\mathrm{e}^{(2-10)}$.

O diagnóstico é feito clinicamente, observando-se as limitações nos movimentos do pescoço, a elevação do ombro no lado do músculo contraturado e a posição da cabeça em inclinação ipsilateral e rotação contralateral ${ }^{(9-11)}$. Um nódulo pode estar presente na porção média do músculo esternocleidomastoideo em aproximadamente $20 \%$ dos pacientes ${ }^{(2)}$. Com muita freqüência, este nódulo é detectado entre dez e 14 dias de vida e pode crescer durante duas a quatro semanas, até atingir o tamanho aproximado de uma amêndoa, quando então começa a regredir e pode desaparecer completamente até o oitavo mês de vida ${ }^{(10-12)}$.

Devido ao encurtamento muscular unilateral, a criança com TC prefere dormir na posição prona, com o lado afetado para baixo. Tal posição provoca pressão assimétrica no crânio e nos ossos faciais em desenvolvimento ${ }^{(4)}$. Esta pressão constante na cabeça pode levar a um remodelamento nos ossos da face e resultar em hemihipoplasia facial ou em plagiocefalia ${ }^{(13-15)}$.

O diagnóstico diferencial é feito com outros tipos de torcicolos adquiridos. Os torcicolos adquiridos podem ser decorrentes de problemas oculares, artrite reumatóide devido à subluxação nas duas primeiras vértebras cervicais $(\mathrm{C} 1$ e C2) ou cisto branquial ${ }^{(10-1)}$. O uso de exames subsidiários, como a ressonância magnética, para o diagnóstico diferencial não tem se mostrado efetivo ${ }^{(16)}$.

Existem diversas formas de abordagem do TC, não havendo uma uniformização da sistemática terapêutica ${ }^{(17)}$. Entretanto, o tratamento inicial de escolha é clínico, por meio de fisioterapia ${ }^{(15,18)}$. Com o tratamento fisioterapêutico, $90^{(11)}$ a $95 \%^{(18)}$ das crianças melhoram antes do primeiro ano de vida e $97 \%$ dos pacientes melhoram se o tratamento for iniciado antes dos primeiros seis meses ${ }^{(2)}$. Quando o tratamento fisioterapêutico é tardio, os pacientes podem apresentar complicações como escolioses cervicais e/ou torácicas compensatórias e dores crônicas ${ }^{(14-19)}$.

Uma vez feito o diagnóstico e iniciado programa fisioterapêutico, existe a necessidade de complementar o tratamento com cuidados domiciliares, pois a realização diária e constante dos exercícios melhora o $\mathrm{TC}^{(18)}$. Os pais devem ser orientados e encorajados a participar, realizando os exercícios propostos, e a quantidade de sessões pode influenciar o tempo de resolução da doença. Assim, o tratamento intensivo pode ter melhor resolução e durar menos tempo ${ }^{(2-20)}$.

O estudo da evolução do tratamento fisioterapêutico de crianças com TC permitirá melhorar a programação do tratamento e orientar a família quanto aos cuidados domiciliares. O presente trabalho tem por objetivo avaliar, em 47 pacientes portadores de TC, a evolução clínica da doença e comparar a eficácia do tratamento fisioterapêutico intensivo realizado diariamente com fisioterapia especializada semanal.

\section{Métodos}

Este estudo foi aprovado pela Comissão de Ética e Pesquisa Humana da Faculdade de Ciência Médicas da Universidade Estadual de Campinas (FCM-Unicamp). No período compreendido entre janeiro de 1995 e dezembro de 2003, foram atendidos 47 pacientes com TC no Ambulatório de Fisioterapia e Cirurgia Pediátrica do Hospital de Clínicas da Universidade Estadual de Campinas (HC-Unicamp).

Os prontuários foram analisados retrospectivamente para a coleta das seguintes informações:

a) antecedentes maternos: idade, tipo de parto, antecedentes gestacionais;

b) antecedentes pessoais da criança: sexo, data de nascimento, idade ao diagnóstico, ser ou não primogênito, cor e doenças associadas; 
c) achados clínicos: distúrbio visual, hemihipoplasia da face, existência ou não de rotação cervical, presença ou ausência de nódulo intramuscular, localização do nódulo no pescoço e complicações evolutivas;

d) tratamento: data da primeira consulta, data da última consulta, data do início da melhora, período entre o diagnóstico e a melhora do estado clínico, tratamento intensivo, tratamento mínimo, necessidade ou não de intervenção cirúrgica, tempo total de tratamento e cura.

Foram incluídos no estudo recém-nascidos ou lactentes de até seis meses de idade, sem doenças neurológicas, com diagnóstico de TC. Foram excluídas crianças encaminhadas ao ambulatório da Unicamp com mais de seis meses de idade, com doenças neurológicas ou que foram submetidas a tratamento cirúrgico. O diagnóstico de TC foi clínico, a partir da observação da posição e da palpação de nódulo muscular.

A classificação do tratamento em intensivo ou mínimo foi feita de acordo com a adesão e a freqüência de sessões fisioterapêuticas. O tratamento intensivo consistiu na realização diária de sessões fisioterapêuticas, contando com a adesão dos pais para a aplicação dos exercícios e dos cuidados domiciliares. O fisioterapeuta acompanhou as crianças no ambulatório, em sessões semanais ou quinzenais, de acordo com a disponibilidade do paciente para retornar. Nessas sessões, os pais foram treinados e orientados para continuar o tratamento em casa. O tratamento foi realizado de acordo com Leung e Leung ${ }^{(19)}$ e consistiu em analgesia com calor local superficial, massoterapia e alongamentos para o esternocleidomastoideo, com a criança em decúbito dorsal. Com uma das mãos apoiando o ombro ipsilateral ao músculo contraído, aplicou-se, com a outra mão, uma flexão lateral da cabeça em direção ao ombro contralateral. Em seguida, repetiu-se este alongamento, tentando realizar rotação cervical simultânea à flexão lateral para o lado contraído. Cada alongamento foi realizado cinco vezes, permanecendo por dez segundos em cada posição. Após o término do atendimento, os pais foram orientados para que os exercícios domiciliares fossem feitos duas vezes ao dia, de acordo com Emery ${ }^{(15)}$. Em complementação aos exercícios domiciliares, foi solicitado aos pais que observassem e corrigissem o posicionamento da cabeça da criança durante o sono, o transporte e a amamentação, de modo a favorecer o alongamento passivo do músculo contraído e, também, solicitou-se que intensificassem o tratamento com estímulos visuais e sonoros, procurando favorecer a movimentação ativa do pescoço de forma lúdica ${ }^{(11)}$.
O tratamento mínimo correspondeu a sessões semanais ou quinzenais no Ambulatório de Fisioterapia, sem a adesão domiciliar diária. Este tratamento foi direcionado às famílias que não efetuaram o tratamento em casa por dificuldades no aprendizado, insegurança ou impossibilidade de vir ao hospital regularmente para receber orientações a respeito do tratamento fisioterapêutico.

$\mathrm{O}$ critério adotado para determinar a cura do TC e alta da fisioterapia consistiu na avaliação clínica, na qual o fisioterapeuta evidenciou a ausência de nódulo à palpação, a cabeça posicionada na linha média, com livre amplitude nos movimentos cervicais.

Os dados dos dois grupos foram comparados por meio do teste do qui-quadrado e do teste exato de Fisher, com nível de significância fixado em $p<0,05$.

\section{Resultados}

Os dados referentes aos antecedentes maternos, antecedentes pessoais, apresentação dos dados clínicos e do tratamento estão apresentados na Tabela 1 . Todas as crianças nasceram a termo. A cura foi observada em $77 \%$ das crianças do grupo de tratamento mínimo e em $100 \%$ das crianças do grupo de tratamento intensivo. O total de complicações evolutivas e o tempo para a cura foram significantemente menores nos pacientes submetidos ao tratamento intensivo $(p=0,02)$. Não foi observada diferença na comparação entre as demais variáveis.

\section{Discussão}

O TC consiste em uma alteração músculo-esquelética que acomete principalmente neonatos e lactentes, sendo o esternocleidomastoideo o principal músculo envolvido. Os pacientes portadores de TC apresentam uma inclinação da cabeça ipsilateral ao esternocleidomastoideo contraído e rotação cervical para o lado contralateral, podendo ou não estar presente um nódulo na porção média do músculo ${ }^{(10,15-22)}$.

Embora exista na literatura grande número de trabalhos que demonstrem a eficácia da fisioterapia para o tratamento de crianças com $\mathrm{TC}^{(9,15-22)}$, pouco foi descrito sobre a freqüência dos exercícios e do acompanhamento ambulatorial para se obter melhores resultados em menor tempo de tratamento. No entanto, o diagnóstico precoce e o tratamento intensivo fisioterapêutico parecem ser uma abordagem eficaz para crianças menores de um ano de idade ${ }^{(23)}$. 
Tabela 1 - Dados maternos, neonatais e referentes ao tratamento das 47 crianças com torcicolo congênito

\begin{tabular}{|c|c|c|c|c|}
\hline & $\begin{array}{c}\text { Geral } \\
(n=47)\end{array}$ & $\begin{array}{c}\text { Tratamento } \\
\text { mínimo } \\
(n=13)\end{array}$ & $\begin{array}{c}\text { Tratamento } \\
\text { intensivo } \\
(n=34)\end{array}$ & $\begin{array}{c}\text { Mínimo versus } \\
\text { intensivo } \\
p\end{array}$ \\
\hline Idade materna (anos) & $24 \pm 6$ & $25 \pm 7$ & $23 \pm 6$ & $>0,05$ \\
\hline Primigesta & $35(75 \%)$ & $10(77 \%)$ & $25(74 \%)$ & $>0,05$ \\
\hline Parto & & & & $>0,05$ \\
\hline Normal & $21(45 \%)$ & $6(46 \%)$ & $15(44 \%)$ & \\
\hline Fórceps & $19(40 \%)$ & $5(39 \%)$ & $14(41 \%)$ & \\
\hline Cesárea & $7(15 \%)$ & $2(15 \%)$ & $5(15 \%)$ & \\
\hline Sexo masculino & $25(53 \%)$ & $7(54 \%)$ & $18(53 \%)$ & $>0,05$ \\
\hline Peso ao nascer (g) & $2899 \pm 304$ & $2870 \pm 281$ & $2910 \pm 312$ & $>0,05$ \\
\hline Dias ao diagnóstico & $67 \pm 40$ & $65 \pm 40$ & $67 \pm 39$ & $>0,05$ \\
\hline Cor branca & $42(89 \%)$ & $12(92 \%)$ & $5(15 \%)$ & $>0,05$ \\
\hline Doenças associadas & & & & $>0,05$ \\
\hline Luxação de quadril & $4(8 \%)$ & $2(15 \%)$ & $2(6 \%)$ & \\
\hline Fratura de clavícula & $2(4 \%)$ & $2(15 \%)$ & 0 & \\
\hline Pé torto congênito & $1(2 \%)$ & 0 & $1(3 \%)$ & \\
\hline Achados clínicos & & & & $>0,05$ \\
\hline Hemihipoplasia facial & $6(13 \%)$ & $3(23 \%)$ & $3(9 \%)$ & \\
\hline Rotação cervical & $14(30 \%)$ & $5(39 \%)$ & $9(26 \%)$ & \\
\hline Nódulo & $46(98 \%)$ & $12(92 \%)$ & $34(100 \%)$ & \\
\hline Lateralidade & & & & $>0,05$ \\
\hline Direita & $25(53 \%)$ & $9(69 \%)$ & $16(47 \%)$ & \\
\hline Esquerda & $22(47 \%)$ & $4(31 \%)$ & $18(53 \%)$ & \\
\hline Complicações evolutivas & & & & 0,02 \\
\hline Estrabismo & $1(2 \%)$ & $1(8 \%)$ & $1(3 \%)$ & \\
\hline Hemi-hipoplasia facial & $2(4 \%)$ & $2(15 \%)$ & 0 & \\
\hline Escoliose & $2(4 \%)$ & $1(8 \%)$ & 0 & \\
\hline Início da melhora (dias) & $30 \pm 41$ & $56 \pm 70$ & $20 \pm 14$ & $>0,05$ \\
\hline Cura (dias) & $90 \pm 64$ & $132 \pm 95$ & $74 \pm 38$ & 0,02 \\
\hline
\end{tabular}

No Brasil, os recursos são precários e existe muita dificuldade na adesão ao tratamento fisioterapêutico diário por grande parte das famílias atendida nos serviços públicos universitários. Devido a tal situação, pôde-se observar, nesse estudo, que algumas famílias aderiram completamente ao tratamento, realizando o tratamento domiciliar, e outras parcialmente, comparecendo apenas às sessões semanais no Ambulatório de Fisioterapia.

Apesar de o TC ser uma doença bem caracterizada e haver conseqüências estéticas desfavoráveis para crianças não tratadas precocemente, ainda não há um consenso sobre sua etiologia. Estudos tentam associar estatisticamente a causa do TC com o histórico obstétrico. Uma das possíveis causas e principal fator da patogênese do TC pode ser o trauma no parto ${ }^{(24)}$.

Hsieh $e t$ al ${ }^{(25)}$ constataram uma incidência de $2 \%$ de TC em partos traumáticos e de $0,3 \%$ em partos não traumáticos. No presente estudo, em $40 \%$ dos casos o parto foi fórceps. MacDonald ${ }^{(26)}$ observou, em avaliação de 50 crianças portadoras de TC, que $24 \%$ delas fizeram uso de fórceps durante o parto.

Outro achado importante associado ao TC e relacionado a traumas durante o parto é o alto índice de crianças nascidas de mães primigestas. Das 47 crianças deste estudo, $75 \%$ das mães eram primigestas. Esta observação é similar ao referido 
por MacDonald ${ }^{(26)}$, que encontrou $50 \%$ de mães primigestas em crianças com TC, e por Ling e Low ${ }^{(27)}$, que mostraram $48 \%$ de primigestas em sua casuística.

Nesse estudo, foram registradas algumas doenças associadas ao TC, como a luxação congênita de quadril em $8 \%$ dos casos, fratura de clavícula em $4 \%$ e pé torto congênito em $2 \%$ das crianças. De maneira similar à relatada na literatura, a luxação congênita de quadril foi a doença mais associada ao $\mathrm{TC}^{(1)}$. Morrison e MacEwen ${ }^{(28)}$ observaram incidência de displasias de quadril em aproximadamente $14 \%$ dos pacientes com TC. Walsh e Morrisy ${ }^{(29)}$ indicam a investigação de displasia de quadril sempre que há TC, visto que tais autores encontraram associação entre ambas em $8 \%$ dos casos.

A ocorrência de TC em crianças nascidas de partos cesáreas é outro achado importante já relatado por MacDonald ${ }^{(26)}$, que observou incidência de $24 \%$ em filhos de mães submetidas a cesárea, enquanto Ling e Low ${ }^{(27)}$ relataram incidência de $9 \%$. No presente estudo, com 47 crianças portadoras de TC, 15\% nasceram de parto cesáreo, sugerindo que a causa do TC pode não estar associada exclusivamente a traumas durante o parto.

Assim como relatado na literatura ${ }^{(9)}$, no presente estudo não houve diferença estatística quanto à distribuição por sexo, sendo 25 crianças do sexo masculino $(53,2 \%)$ e 22 do feminino $(46,8 \%)$.

$\mathrm{O}$ achado clínico mais freqüente neste estudo foi a presença de nódulo intramuscular, em 97,9\% dos casos. Este resultado difere do registrado por Morrison e MacEwen ${ }^{(28)}$, que encontraram nos 232 pacientes analisados maior freqüência de rotação cervical (81\%). Segundo Chandler e Altenberger ${ }^{(10)}$, o nódulo pode estar presente no nascimento ou se manifestar entre o décimo e o $14^{\circ}$ dia de vida, aumentando de tamanho por 14 a 28 dias.

A idade média ao diagnóstico de TC, no presente estudo, foi de aproximadamente 50 dias, enquanto, na população analisada por Morrison e MacEwen ${ }^{(28)}$, aproximadamente metade dos pacientes tinha menos de 21 dias de vida. Acredita-se que o diagnóstico mais tardio possa estar ligado à não atenção ao nódulo clinicamente presente por parte do médico ou ao acesso tardio à rede básica de atendimento de puericultura. No entanto, os resultados do presente estudo estão de acordo com Chandler e Altenberg ${ }^{(10)}$, que observaram manifestações clínicas mais evidentes quando o nódulo não foi verificado precocemente e o diagnóstico foi tardio.

Vários métodos têm sido usados no tratamento do TC e muitos autores registraram bons resultados com o tratamento fisioterapêutico ${ }^{(15-30)}$. Nesta pesquisa, observou-se cura em todos os pacientes que seguiram o tratamento até o final e apenas três pacientes perderam o seguimento ambulatorial. O tempo de cura do TC foi menor no grupo de pacientes que aderiu ao tratamento intensivo, comparado ao tratamento mínimo. O tempo médio para a resolução do quadro foi de 74 dias (30 a 180) no grupo de tratamento intensivo e de 130 dias (30 a 270) no grupo de tratamento mínimo.

Leung e Leung ${ }^{(19)}$, em estudo com 206 pacientes, registraram $90 \%$ de cura em um tempo médio de 120 dias de tratamento, com alongamentos duas vezes por semana e correções ativas do pescoço dia-a-dia. Tal estudo não descreveu o intervalo em que as crianças retornavam ao ambulatório para o acompanhamento com o fisioterapeuta.

Emery ${ }^{(15)}$ verificou cura em 100 das 101 crianças tratadas com fisioterapia. $\mathrm{O}$ tratamento consistia de alongamentos diários realizados pelos pais, após serem treinados pelo fisioterapeuta que acompanhava as crianças em ambulatório, com retorno a cada duas semanas no início e, mensalmente, até o término do tratamento. Este tratamento durou, em média, 140 dias, superior ao observado no presente estudo.

Os resultados desta pesquisa mostraram que a fisioterapia atua no sentido de evitar uma intervenção cirúrgica face ao diagnóstico do TC, além de ser um tratamento eficiente e não traumático ao paciente ${ }^{(17)}$. O tempo para obter a cura depende da intensidade e freqüência de realização da fisioterapia. O tratamento assistido e assumido pelos pais ou responsáveis pela criança é outro fator importante na obtenção da cura em tempo reduzido. A conscientização e comprometimento dos familiares no tratamento diário é um fator importante, que deve ser explicado pelo fisioterapeuta durante a orientação dos exercícios.

É importante ressaltar que a resposta ao tratamento fisioterapêutico também foi positiva para o grupo do tratamento mínimo. Embora significativamente mais demorada do que no grupo de tratamento intensivo, a cura foi obtida em dez (77\%) crianças submetidas apenas às sessões semanais de fisioterapia, demorando 130 dias até a resolução do quadro. Tais crianças também apresentaram maior número de complicações evolutivas. Os resultados comprovam que a fisioterapia pode ser indicada como método inicial de tratamento para o TC, mesmo nos casos em que os familiares não tenham condições de aderir ao tratamento domiciliar intensivo. Tais resultados obtidos com o tratamento mínimo foram similares aos obtidos por Emery ${ }^{(15)}$, que relatou a importância da fisioterapia no tratamento de crianças portadoras de TC.

Apesar das limitações inerentes a um estudo retrospectivo, procurou-se enfatizar o diagnóstico precoce, a importância 
e eficácia do tratamento fisioterapêutico intensivo ou mínimo para o TC, indicando aos profissionais que cuidam de neonatos, como os fisioterapeutas, pediatras e cirurgiões pediátricos, a importância do acompanhamento domiciliar para a resolução do $\mathrm{TC}$ em tempo reduzido, com redução da ansiedade dos pais.

\section{Referências bibliográficas}

1. Wei JL, Schwartz KM, Weaver AL, Orvidas LJ. Pseudotumor of infancy and congenital muscular torticollis: 170 cases. Laryngoscope 2001;111:688-95.

2. Cheng JC, Au AW. Infantile torticollis: A review of 624 cases. J Pediatr Orthop 1994;14:802-8

3. Hollier L, Kim J, Grayson BH, McCarthy JG. Congenital muscular torticollis and the associated craniofacial changes. Plast Reconstr Surg 2000;105: 827-35.

4. Davids JR, Wenger DR, Mubarak SJ. Congenital muscular torticollis: sequela of intrauterine or perinatal compartment syndrome. J Pediatr Orthop 1993;13:141-7.

5. Burstein FD, Cohen SR. Endoscopic surgical treatment for congenital muscular torticollis. Plast Reconstr Surg 1998;101:20-4.

6. Nové-Josserand G, Viannay C. Pathogénie du torticollis congénital. Rev d'Orthop 1906;7:397.

7. Kempf F. Üeber ursache und behandlung des caput obstipum musculare. Langenbecks Arch Chir 1904;73:351-87.

8. Middleton DS. The pathology of congenital torticollis. Br J Surg 1930;18:188-204.

9. Wolfort FG, Kanter MA, Miller LB. Torticollis. Plast Reconstr Surg 1989;84:682-92.

10. Chandler FA, Altenberg A. Congenital muscular torticollis. JAMA 1944;125:476-83.

11. Chen CE, Ko JY. Surgical treatment of muscular torticollis for patients above 6 years of age. Arch Orthop Trauma Surg 2000;120:149-51.

12. Gonzales J, Ljung BM, Guerry T, Schoenrock LD. Congenital torticollis: evaluation by fine-needle aspiration biopsy. Laryngoscope 1989;99:651-4.

13. Dunne KB, Clarren SK. The origin of prenatal and postnatal deformities. Pediatr Clin North Am 1986;33:1277-97.

14. Bredenkamp JK, Hoover LA, Berke GS, Shaw A. Congenital muscular torticollis. A spectrum of disease. Arch Otolaryngol Head Neck Surg 1990;116:212-6.

15. Emery $\mathrm{C}$. The determinants of treatment duration for congenital muscular torticollis. Phys Ther 1994;74:921-9.

16. Parikh SN, Crawford $\mathrm{AH}$, Choudhury S. Magnetic resonance imaging in the evaluation of infantile torticollis. Orthopedics 2004;27:509-15.

\section{Agradecimentos}

À Camila Isabel da S. Santos, fisioterapeuta do Hospital de Clínicas da Universidade Estadual de Campinas (HCUnicamp), amiga e colega de trabalho, pela receptividade e cooperação neste manuscrito.
17. van Vlimmeren LA, Helders PJ, van Adrichem LN, Engelbert RH. Torticollis and plagiocephaly in infancy: therapeutic strategies. Pediatr Rehabil 2006;9:40-6.

18. Cheng JC, Chen TM, Tang SP, Shum SL, Wong MW, Metreweli C. Snapping during manual stretching in congenital muscular torticollis. Clin Orthop Relat Resp 2001:237-44.

19. Leung YK, Leung PC. The efficacy of manipulative treatment for sternomastoid tumours. J Bone Joint Surg Br 1987;69:473-8.

20. Luther BL. Congenital muscular torticollis. Orthop Nurs 2002;21:21-7.

21. Shepherd RB. Fisioterapia em pediatria. $3^{\text {a }}$ ed. São Paulo: Livraria Santos Editora; 1995.

22. Cheng JC, Tang SP, Chen TM, Wong MW, Wong EM. The clinical presentation and outcome of treatment of congenital muscular torticollis in infants-a study of 1086 cases. J Pediatr Surg 2000;35:1091-6.

23. Do TT. Congenital muscular torticollis: current concepts and review of treatment. Curr Opin Pediatr 2006;18:26-9

24. Coventry MB, Harris LE. Congenital muscular torticollis in infancy: some observations regarding treatment. J Bone Joint Surg Am 1959;41-A:815-22.

25. Hsieh YY, Tsai FJ, Lin CC, Chang FC, Tsai CH. Breech deformation complex in neonates. J Reprod Med 2000;45:933-5.

26. MacDonald D. Sternomastoid tumour and muscular torticollis. J Bone Joint Surg $\mathrm{Br}$ 1969;51:432-43.

27. Ling CM, Low YS. Sternomastoid tumor and muscular torticollis. Clin Orthop Relat Res 1972;86:144-50.

28. Morrison DL, MacEwen GD. Congenital muscular torticollis: observations regarding clinical findings, associated conditions, and results of treatment. J Pediatr Orthop 1982;2:500-5.

29. Walsh JJ, Morrissy RT. Torticollis and hip dislocation. J Pediatr Orthop 1998;18:219-21.

30. Canale ST, Griffin DW, Hubbard CN. Congenital muscular torticollis. A longterm follow-up. J Bone Joint Surg Am 1982;64:810-6. 